Document downloaded from:

http://hdl.handle.net/10251/120655

This paper must be cited as:

Esteso, A.; Alemany Díaz, MDM.; Ortiz Bas, A.; Guyon, C. (2018). A Collaborative Model to Improve Farmers' Skill Level by Investments in an Uncertain Context. IFIP Advances in Information and Communication Technology. 534:590-598. https://doi.org/10.1007/978-3319-99127-6_51

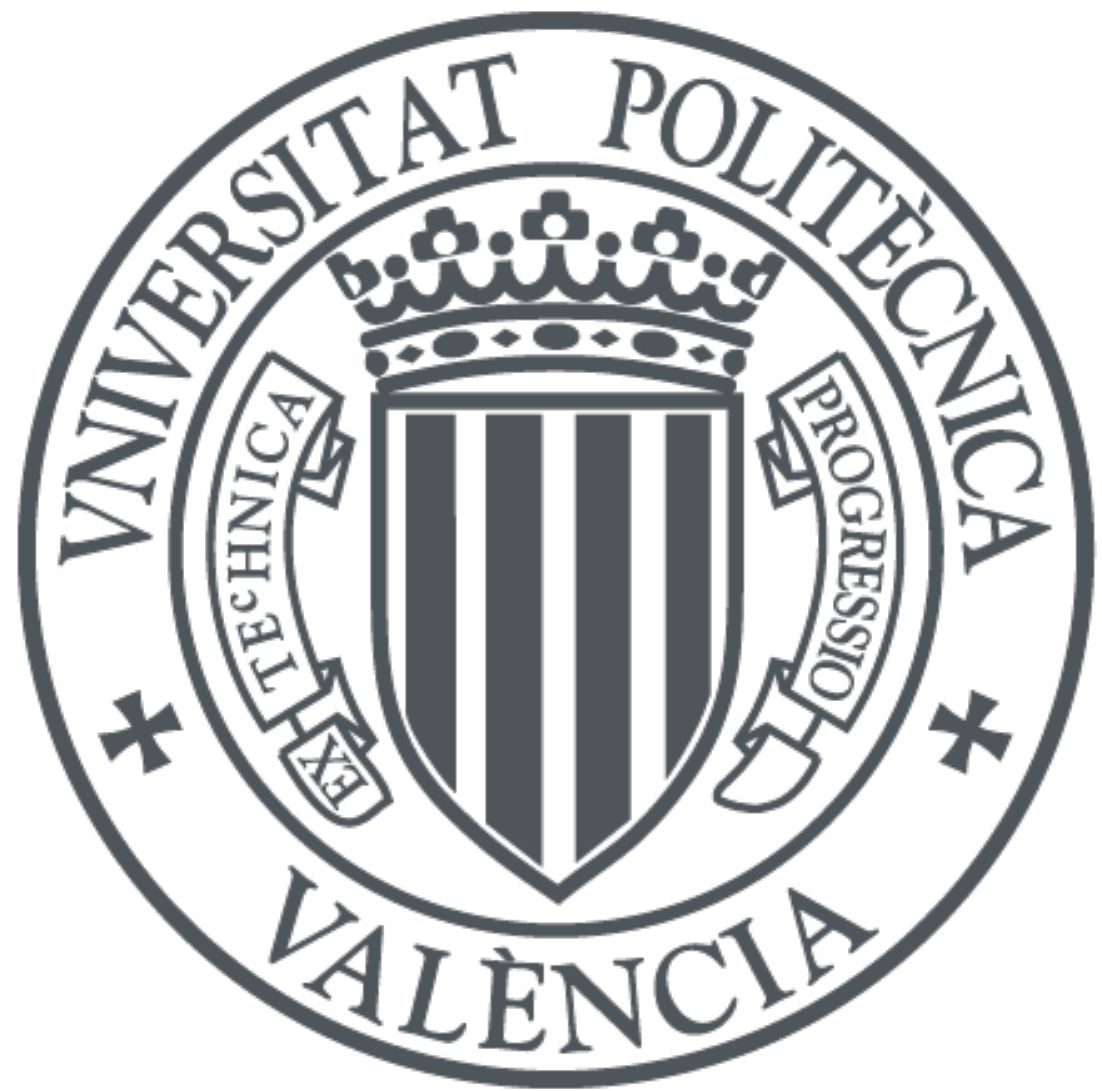

The final publication is available at

http://doi.org/10.1007/978-3-319-99127-6_51

Copyright Springer

Additional Information 


\title{
A Collaborative Model to Improve Farmers' Skill Level by Investments in an Uncertain Context
}

\author{
Ana Esteso $^{1}, \mathrm{MME}$ Alemany ${ }^{1}$, Ángel Ortiz ${ }^{1}$, and Cecile Guyon ${ }^{2}$, \\ ${ }^{1}$ Research Centre on Production Management and Engineering (CIGIP). Universitat \\ Politècnica de València. Camino de Vera S/N, 46022 València, Spain; ${ }^{2}$ Bretagne Development \\ Innovation. 1bis Route de Fougères, 35510 Cesson-Sévigne, France \\ aneslva@doctor.upv.es, \{mareva, aortiz\}@cigip.uvp.es, c.guyon@bdi.fr
}

\begin{abstract}
Some small farms are forced to waste a part of their harvests for not reaching the quality standards fixed by consumers. Meanwhile, modern retailers (MR) are interested in selling more quality products to increase their profits. MR could invest in a collaboration program so the small farmers could have access to better technologies and formation to increase the proportion of quality products. Unfortunately, the demand, the quantity of harvest, the proportion of harvest being of quality, and its increase with each investment are uncertain parameters. A fuzzy model considering these uncertainties is proposed to determine the investments that MR should made to maximize the profits of the supply chain in a collaboration context. A method to transform the fuzzy model into an equivalent crisp model and an interactive resolution method are applied.
\end{abstract}

Keywords: Agri-Food Supply Chain; Farmer Skills; Collaboration; Product Quality; Fuzzy Mathematical Programming.

\section{Introduction}

Quality standards imposed by end consumers forces some small farmers to throw away big amounts of products. This fact negatively impacts on the environment and the small farmers economies. If the proportion of quality products (QP) obtained in each harvest could be increased, this problem would be eliminated or mitigated. A high level of collaboration is necessary to ensure the quality of the agri-food products [1].

Recent papers propose models to empower small farmers through modern retailers' investments [2-8], but none of them considers the uncertainty of consumers' demand, quantity harvested, proportion of QP obtained from harvest, nor its improvement with each modern retailers' investment. If uncertainty is not considered, models will obtain solutions only applicable to situations in which all the data is known in advance. This paper aims to fill this gap by adapting the model [2] to the uncertain nature of these parameters. Methods to convert the fuzzy model into an equivalent crisp model [9] and a to select the best solution to implement in the AFSC [10] are employed.

The paper is structured as follows. Section 2 describes the problem addressed. Section 3 formulates the fuzzy model. Section 4 explains the methods used to solve the model and to select the solution to be implemented. In Section 5 these methods are applied. Conclusions and future research lines are drawn in Section 6. 


\section{Problem Description}

The AFSC is responsible for the production and distribution of vegetables. It is comprised by small farmers (SF), farmer cooperatives (FC), modern retailers (MR), and consumer markets $(\mathrm{CM})$. End consumers require vegetables with a minimum quality standard, however not all vegetables harvested by SF meet these standards. In fact, the quantity of harvest and the proportion of QP obtained in each harvest are uncertain. Once harvest is made, FCs classify the products into QP and non-quality products (NQP). FCs sell QP to MR, which are responsible of the QP distribution to CM. To reduce wastes, NQP are directly sold to $\mathrm{CM}$ at a very low price.

To increase the AFSC profits, more demand need to be covered with QP. For that, MR and SF can establish a collaboration program (CP). In this CP, MR would choose one or more SF and would give them funds with the objective to improve the quality of products. SF should use these funds to acquire new technologies, machineries and/or training. This will increase the proportion of QP to be harvested.

The CP sets three skill levels to which SF can belong according to the proportion of QP obtained in each harvest. When a MR funds one SF, the latter can improve the proportion of QP to be harvested and therefore SF can move up from one skill level to another. However, the improvement of the QP proportion is not known in advance to the fund application. MRs' investments cannot exceed the available budget for the CP.

A fuzzy model for deciding the investments to carry out to maximize the AFSC profits is proposed. The quantity of harvest, the proportion of it being of quality, the improvement of such proportion with each investment, and the demand are uncertain.

\section{Fuzzy Model Formulation}

The nomenclature employed to formulate the model is exposed in Table 1, where $v$ refers to vegetables, $c$ to the vegetables quality, $i$ to SF, $j$ to $\mathrm{FC}, k$ to $\mathrm{MR}, m$ to $\mathrm{CM}, t$ to periods of time, and $F C_{i}$ to the set of SFs that belong to a particular FC $j$. The fuzzy model based on [2] can be presented as follows:

$$
\begin{aligned}
\max Z=\sum_{v} \sum_{c} \sum_{i} & \sum_{j \in F C_{i}} \sum_{m} \sum_{t}\left(\sum_{k} Q_{i j k m}^{v c t}+q m_{i j m}^{v c t}\right) \cdot p_{i j m}^{v c t} \\
& -\sum_{v} \sum_{c} \sum_{i} \sum_{j \in F C_{i}} \sum_{t} q_{i j}^{v c t} \cdot\left(d i j_{i j}^{v t}+r_{i j}^{v t}\right) \\
& -\sum_{v} \sum_{c} \sum_{i} \sum_{j \in F C_{i}} \sum_{k} \sum_{t} q k_{i j k}^{v c t} \cdot d j k_{j k}^{v t} \\
& -\sum_{v} \sum_{c} \sum_{i} \sum_{j \in F C_{i}} \sum_{m} \sum_{t} q m_{i j m}^{v c t} \cdot d j m_{j m}^{v t} \\
& -\sum_{v} \sum_{c} \sum_{i} \sum_{j \in F C_{i}} \sum_{k} \sum_{m} \sum_{t} Q_{i j k m}^{v c t} \cdot d k m_{k m}^{v t} \\
& -\sum_{v} \sum_{t}\left(\sum_{i} w_{i}^{v t}+\sum_{m} r d_{m}^{v t}\right) \cdot p c^{v t}-\sum_{i} \sum_{j \in F C_{i}} \sum_{t} F_{i j}^{t} \cdot h_{i j}^{t}
\end{aligned}
$$


Table 1. Nomenclature

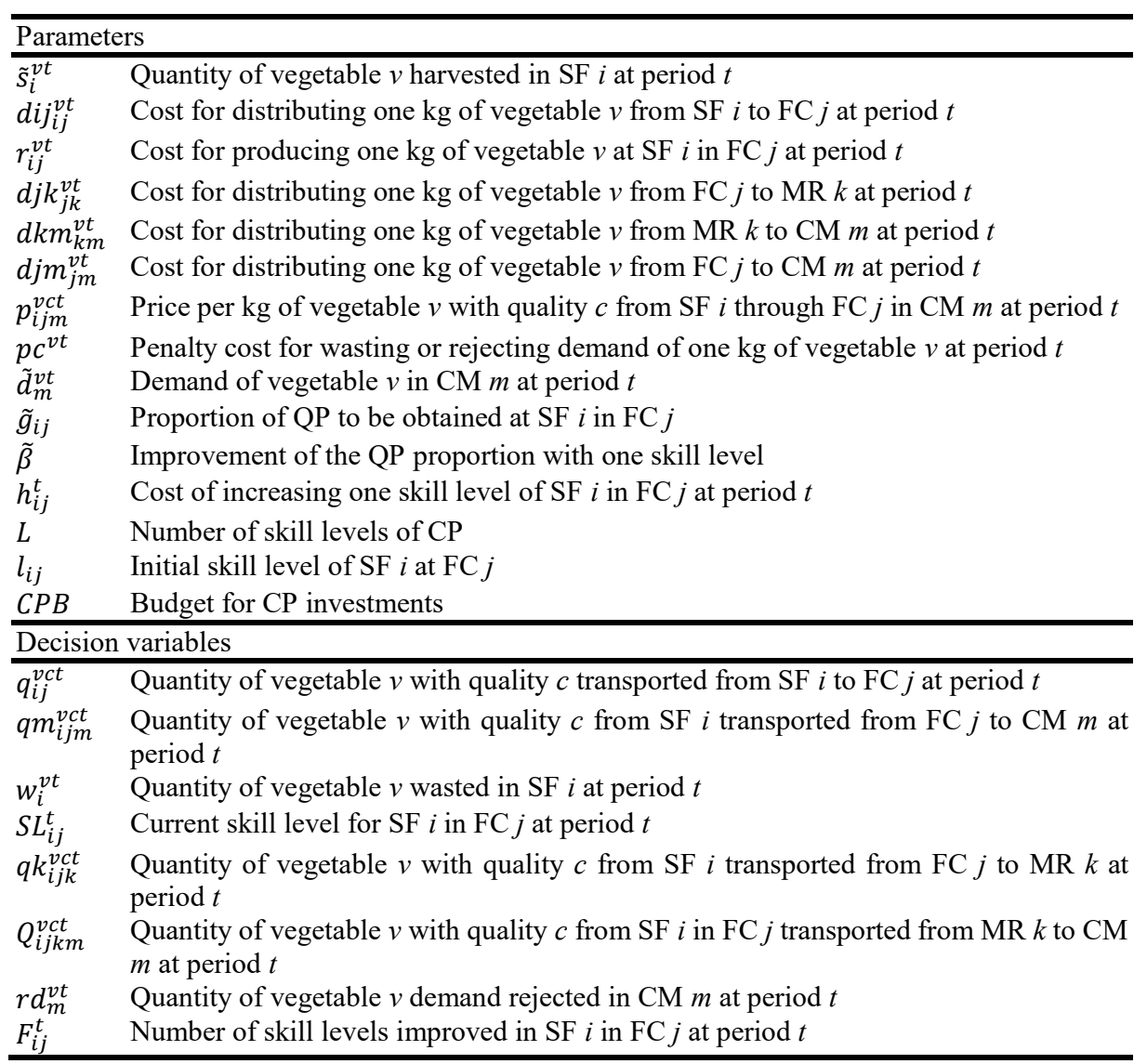

Subject to:

$$
\begin{gathered}
\tilde{s}_{i}^{v t}=\sum_{j \in F C_{i}} \sum_{c} q_{i j}^{v c t}+w_{i}^{v t} \quad \forall i, v, t \\
q_{i j}^{v c t} \leq \tilde{s}_{i}^{v t} \cdot\left(\tilde{g}_{i j}+\tilde{\beta} \cdot S L_{i j}^{t}\right) \quad \forall i, j \in F C_{i}, v, c=1, t \\
q_{i j}^{v c t} \leq \tilde{s}_{i}^{v t} \cdot\left(1-\tilde{g}_{i j}-\tilde{\beta} \cdot S L_{i j}^{t}\right) \quad \forall i, j \in F C_{i}, v, c=2, t \\
q_{i j}^{v c t}=\sum_{k} q k_{i j k}^{v c t} \quad \forall i, j \in F C_{i}, v, c=1, t \\
q_{i j}^{v c t}=\sum_{k} q k_{i j k}^{v c t} \quad \forall i, j \in F C_{i}, v, c=2, t \\
q m_{i j m}^{v c t}=0 \quad \forall i, j \in F C_{i}, v, m, c=1, t
\end{gathered}
$$




$$
\begin{gathered}
q m_{i j m}^{v c t}=0 \quad \forall i, j \in F C_{i}, v, m, c=1, t \\
q k_{i j k}^{v c t}=\sum_{m} Q_{i j k m}^{v c t} \quad \forall i, j \in F C_{i}, k, v, c, t \\
Q_{i j k m}^{v c t} \leq q k_{i j k}^{v c t} \quad \forall i, j \in F C_{i}, k, m, v, c, t \\
\sum_{i} \sum_{j \in F C_{i}} \sum_{c}\left(q m_{i j m}^{v c t}+\sum_{k} Q_{i j k m}^{v c t}\right)+r d_{m}^{v t}=\tilde{d}_{m}^{v t} \quad \forall m, v, t \\
\sum_{i} \sum_{j \in F C_{i}} \sum_{t} F_{i j}^{t} \cdot h_{i j}^{t} \leq C P B \\
\left(\tilde{g}_{i j}+\tilde{\beta} \cdot S L_{i j}^{t}\right) \leq 1 \quad \forall i, j \in F C_{i}, t \\
S L_{i j}^{t}=l_{i j}+\sum_{t_{2}=0}^{t} F_{i j}^{t_{2}} \quad \forall i, j \in F C_{i}, t \\
S L_{i j}^{t}=L \quad \forall i, j \in F C_{i}, t \\
F_{i j}^{t}, S L_{i j}^{t} \quad I N T E G E R \\
q_{i j}^{v c t}, q k_{i j k}^{v c t}, q m_{i j m}^{v c t}, Q_{i j k m}^{v c t}, w_{i}^{v t}, r d_{m}^{v t} \\
\text { CONTINUOUS }
\end{gathered}
$$

The model aims to maximize the profits obtained by the whole AFSC (1). For that, profits obtained when selling QP or NQP, as well as costs related to production, distribution, penalties for rejecting demand or wasting products, and investments in the collaboration program are considered.

The product balance at SF is set in constraint (2). Constraints (3) and (4) state the distribution of harvested product between QP and NQP respectively. Constraints (5) to (8) define the product flow between FC, MR and CM, ensuring that QP are only distributed through MR and NQP are directly served to CM. Product balance at MR is set in constraints (9) and (10). Quantity of demand being served and/or rejected is determined in constraint (11). Constraint (12) ensures that investments in the CP do not exceed the available budget for that purpose. The inability to obtain more QP than the quantity of harvested products is defined in constraint (13). Current skill level for each $\mathrm{SF}$ is calculated in constraint (14) and constraint (15) forces it to be lower than or equal to the maximum skill level of the program. Finally, constraint (16) sets the definition of variables.

\section{Solution Method}

First, the methodology proposed by Jiménez et al. [9] to transform a fuzzy model into an equivalent auxiliary crisp model is employed. The auxiliary MILP crisp model is comprised by the same objective function and constraints that the fuzzy model except 
for constraints (2-4), (11) and (13) that are replaced by constraints (17-23). We recommend readers to consult original source [9] for more information of this approach.

$$
\begin{aligned}
& {\left[\frac{\alpha}{2} \cdot\left(\frac{s_{i}^{v t 1}+s_{i}^{v t 2}}{2}\right)+\left(1-\frac{\alpha}{2}\right) \cdot\left(\frac{s_{i}^{v t 2}+s_{i}^{v t 3}}{2}\right)\right] \geq \sum_{j \in F C_{i}} \sum_{c} q_{i j}^{v c t}+w_{i}^{v t} \quad \forall i, v, t} \\
& {\left[\frac{\alpha}{2} \cdot\left(\frac{s_{i}^{v t 2}+s_{i}^{v t 3}}{2}\right)+\left(1-\frac{\alpha}{2}\right) \cdot\left(\frac{s_{i}^{v t 1}+s_{i}^{v t 2}}{2}\right)\right] \leq \sum_{j \in F C_{i}} \sum_{c} q_{i j}^{v c t}+w_{i}^{v t} \quad \forall i, v, t} \\
& q_{i j}^{v c t} \leq\left[\alpha \cdot\left(\frac{s_{i}^{v t 1}+s_{i}^{v t 2}}{2}\right)+(1-\alpha) \cdot\left(\frac{s_{i}^{v t 2}+s_{i}^{v t 3}}{2}\right)\right]+\left(\left[\alpha \cdot\left(\frac{g_{i j}^{1}+g_{i j}^{2}}{2}\right)+\right.\right. \\
& \left.\left.(1-\alpha) \cdot\left(\frac{g_{i j}^{2}+g_{i j}^{3}}{2}\right)\right]+\left[\alpha \cdot\left(\frac{\beta^{1}+\beta^{2}}{2}\right)+(1-\alpha) \cdot\left(\frac{\beta^{2}+\beta^{3}}{2}\right)\right] \cdot S L_{i j}^{t}\right) \\
& \forall i, j \in F C_{i}, v, c=1, t \\
& q_{i j}^{v c t} \leq\left[\alpha \cdot\left(\frac{s_{i}^{v t 1}+s_{i}^{v t 2}}{2}\right)+(1-\alpha) \cdot\left(\frac{s_{i}^{v t 2}+s_{i}^{v t 3}}{2}\right)\right]+\left(1-\left[\alpha \cdot\left(\frac{g_{i j}^{1}+g_{i j}^{2}}{2}\right)+\right.\right. \\
& \left.\left.(1-\alpha) \cdot\left(\frac{g_{i j}^{2}+g_{i j}^{3}}{2}\right)\right]+\left[\alpha \cdot\left(\frac{\beta^{1}+\beta^{2}}{2}\right)+(1-\alpha) \cdot\left(\frac{\beta^{2}+\beta^{3}}{2}\right)\right] \cdot S L_{i j}^{t}\right) \\
& \forall i, j \in F C_{i}, v, c=2, t \\
& {\left[\alpha \cdot\left(\frac{g_{i j}^{2}+g_{i j}^{3}}{2}\right)+(1-\alpha) \cdot\left(\frac{g_{i j}^{1}+g_{i j}^{2}}{2}\right)\right]+\left[\alpha \cdot\left(\frac{\beta^{2}+\beta^{3}}{2}\right)+\right.} \\
& \left.(1-\alpha) \cdot\left(\frac{\beta^{1}+\beta^{2}}{2}\right)\right] \cdot S L_{i j}^{t} \leq 1 \quad \forall i, j \in F C_{i}, t \\
& \sum_{i} \sum_{j \in F C_{i}} \sum_{c}\left(q m_{i j m}^{v c t}+\sum_{k} Q_{i j k m}^{v c t}\right)+r d_{m}^{v t} \leq\left[\frac{\alpha}{2} \cdot\left(\frac{d_{m}^{v t 1}+d_{m}^{v t 2}}{2}\right)+\right. \\
& \left.\left(1-\frac{\alpha}{2}\right) \cdot\left(\frac{d_{m}^{v t 2}+d_{m}^{v t 3}}{2}\right)\right] \quad \forall m, v, t \\
& \sum_{i} \sum_{j \in F C_{i}} \sum_{c}\left(q m_{i j m}^{v c t}+\sum_{k} Q_{i j k m}^{v c t}\right)+r d_{m}^{v t} \geq\left[\frac{\alpha}{2} \cdot\left(\frac{d_{m}^{v t 2}+d_{m}^{v t 3}}{2}\right)+\right. \\
& \left.\left(1-\frac{\alpha}{2}\right) \cdot\left(\frac{d_{m}^{v t 1}+d_{m}^{v t 2}}{2}\right)\right] \quad \forall m, v, t
\end{aligned}
$$

The grade of feasibility for a particular solution is represented by $\alpha$ that is ranged from 0 to 1 . All the fuzzy parameters follow triangular membership functions: $\tilde{s}_{i j}^{v}=$ $\left(s_{i j}^{v 1}, s_{i j}^{v 2}, s_{i j}^{v 3}\right), \tilde{g}_{i j}=\left(g_{i j}^{1}, g_{i j}^{2}, g_{i j}^{3}\right), \tilde{d}_{m}^{v t}=\left(d_{m}^{v t 1}, d_{m}^{v t 2}, d_{m}^{v t 3}\right), \tilde{\beta}=\left(\beta^{1}, \beta^{2}, \beta^{3}\right)$.

To select the final solution to be implemented in the AFSC, an interactive resolution method proposed by Peidro et al. [10] is followed. This method is comprised by three steps: i) to solve the equivalent auxiliary crisp model for different values of $\alpha$, ii) to determine the satisfaction of decision maker for each $\alpha$ solution, and iii) to select the $\alpha$ solution that better balances its feasibility and the decision maker satisfaction. For more detailed information of this approach, see [10]. 


\section{Implementation and Evaluation}

The model was implemented in MPL ${ }^{\circledR}$ 5.0.6.114 and solved by using Gurobi ${ }^{\mathrm{TM}}$ 7.0.2 Solver. A Microsoft Access Database is used to import input data and save decision variables values. The computer used for solving the model has an Intel ${ }^{\circledR}$ Xeon ${ }^{\circledR}$ CPU E5-2640 v2 with two $2.00 \mathrm{GHz}$ processors, with an installed memory RAM of $32.0 \mathrm{~GB}$ and a 64-bits operating system.

The instance employed for solving the model is the extracted from [2] for the scenario with 120 periods of time and balanced demand-supply except for the fuzzy parameters. Data for $s_{i}^{v t}, d_{m}^{v t}, g_{i j}, \beta[2]$ are used as the central values for the $\tilde{s}_{i}^{v t}, \tilde{d}_{m}^{v t}$, $\tilde{g}_{i j}$, and $\tilde{\beta}$ membership functions. The lower and upper limits for all functions are obtained by decreasing and increasing the central value by $10 \%$.

The model has been solved for different grades of feasibility $\alpha$. To evaluate each solution, two parameters have been selected: the total profits obtained by the whole AFSC (P) and the total quantity of quality products sold (QPS). As a second step, the decision maker specifies the aspiration level $G$ and the tolerance threshold $t t$ that is willing to accept for each evaluation parameter. This information is employed for identifying the membership function (24) that characterizes the satisfaction of the decision maker with each parameter result.

$$
\mu_{\tilde{G}}(z)= \begin{cases}0 & \text { if } z \leq G-t t \\ \lambda \in[0,1] & \text { if } G-t t \leq z \leq G \\ 1 & \text { if } z \geq G\end{cases}
$$

In this case, the decision maker indicates that the aspiration level for $P$ is $85,000 €$ although he would tolerate profits from 75,000 €. Similarly, the decision maker aspirate to sell $360,000 \mathrm{~kg}$ of QP although he would accept to sell at least 260,000 kg of QP. Using this data, the satisfaction grade for each parameter $\left(\mu_{P}\right.$ and $\left.\mu_{Q P S}\right)$ are calculated per solution (24). The global satisfaction level $\Lambda$ for each $\alpha$ solution is determined as a weighted sum of the satisfaction of both evaluation parameters.

The satisfaction of a solution usually increases as the feasibility of the solution decreases. Thus, the solution that better balances the satisfaction degree and the feasibility degree will be selected for its implementation in the AFSC. To determine such balance, an acceptation index $\mathrm{K}$ is calculated for each solution as a weighted sum of the acceptation grade of the feasibility grade $\gamma_{\alpha}$ and the acceptation grade of the satisfaction grade $\gamma_{\Lambda}$. The acceptation grades for $\alpha$ and $\Lambda$ are also determined by the membership function (24). The decision maker determines that the aspiration level for $\alpha$ is 0.7 although he would tolerate a $\alpha$ from 0.5 . Similarly, he will tolerate $\Lambda$ from 0.2 but sets the aspiration level for the $\Lambda$ is 0.6 . Results of the application of this interactive resolution method [10] are presented in Table 2.

The solution obtained with a grade of feasibility equal to 0.6 will be implemented in the AFSC as it has the most elevated acceptation index. In this solution, the MR invest to improve the quantity of QP in $90 \%$ of famers. Some farmers receive just one fund whereas other receive up to three funds. However, only the $67 \%$ of the budget for the CP is used. With these investments, the profits of the whole AFSC increases in a one per cent and the $85 \%$ of demand is fulfilled with QP. Thus, the presented model let 
MR know the number of funds to give to maximize the profits of the whole AFSC, and the specific farmers to which funds need to be given.

Table 2. Interactive Resolution Method Results.

\begin{tabular}{ccccccccc}
\hline$\alpha$ & $P(€)$ & $\mu_{P}$ & $Q P S(\mathrm{~kg})$ & $\mu_{Q P S}$ & $\Lambda$ & $\gamma_{\alpha}$ & $\gamma_{\Lambda}$ & $\mathrm{K}$ \\
\hline 0.0 & 87832.02 & 1.00 & 359113.23 & 1.00 & 1.00 & 0.00 & 1.00 & 0.50 \\
0.1 & 86829.96 & 1.00 & 364062.82 & 1.00 & 1.00 & 0.00 & 1.00 & 0.50 \\
0.2 & 86199.03 & 1.00 & 380605.81 & 1.00 & 1.00 & 0.00 & 1.00 & 0.50 \\
0.3 & 84296.07 & 0.93 & 365272.20 & 1.00 & 0.96 & 0.00 & 1.00 & 0.50 \\
0.4 & 82688.78 & 0.77 & 363798.77 & 1.00 & 0.88 & 0.00 & 1.00 & 0.50 \\
0.5 & 81186.88 & 0.62 & 364107.60 & 1.00 & 0.81 & 0.00 & 1.00 & 0.50 \\
$\mathbf{0 . 6}$ & $\mathbf{7 8 9 1 4 . 0 8}$ & $\mathbf{0 . 3 9}$ & $\mathbf{3 1 6 9 2 5 . 1 4}$ & $\mathbf{0 . 5 7}$ & $\mathbf{0 . 4 8}$ & $\mathbf{0 . 5 0}$ & $\mathbf{0 . 7 0}$ & $\mathbf{0 . 6 0}$ \\
0.7 & $\mathbf{7 5 4 5 2 . 3 8}$ & 0.05 & 276434.09 & 0.16 & 0.10 & 1.00 & 0.00 & 0.50 \\
0.8 & 68686.48 & 0.00 & 247245.82 & 0.00 & 0.00 & 1.00 & 0.00 & 0.50 \\
0.9 & 62100.18 & 0.00 & 234247.68 & 0.00 & 0.00 & 1.00 & 0.00 & 0.50 \\
1.0 & 55528.60 & 0.00 & 228194.37 & 0.00 & 0.00 & 1.00 & 0.00 & 0.50 \\
\hline
\end{tabular}

The solved model counted with 16,441 constraints and 12,000 variables, of which 9,840 were continuous variables and 2,160 were integer variables. The optimal solution has been found for all the $\alpha$ scenarios with an average resolution time of 1.44 seconds.

\section{Conclusions}

A model for empowering small-farmers through funds obtained by modern retailers' investments is proposed. It is considered that the quantity of harvest, the proportion of QP to be obtained from harvest, the improvement of this proportion through the collaboration program and the demand are uncertain parameters. A method to transform the fuzzy model into an equivalent crisp model [9] and an interactive resolution method [10] to select the solution to implement in the AFSC are employed.

To better represent the real behavior of AFSC, the proposed model could be extended by considering more sources of uncertainty existing in AFSC (e.g. economic data) [11]. In addition, the model could be adjusted to represent some real behaviors of consumers. For example, some consumers may not be willing to buy NQP although there is not enough QP to fulfill their demand. In such cases, some demand can be rejected while some NQP can be wasted. The model could also be extended by considering the perishability aspect of the products causing the loss of a proportion of QP and NQP along the entire AFSC. Finally, more realistic managerial and regulatory factors of AFSC as well as other aspects related with the consumers' behavior could be considered to better adjust the proposed model to real AFSC behavior.

Acknowledgments. The first author acknowledges the partial support of the Programme of Formation of University Professors of the Spanish Ministry of Education, Culture, and Sport (FPU15/03595). The other authors acknowledge the 
partial support of Project 691249, "RUC-APS: Enhancing and implementing Knowledge based ICT solutions within high Risk and Uncertain Conditions for Agriculture Production Systems", funded by the EU under its funding scheme H2020MCSA-RISE-2015.

\section{References}

1. Zhao, G., Liu, S., Lopez, C.: A Literature Review on Risk Sources and Resilience Factors in Agri-Food Supply Chains. In: Working Conference on Vitual Enterprises. Springer, Cham, 739-752 (2017).

2. Esteso, A., Alemany, M.M.E., Ortiz, A.: Improving Vegetables Quality in Small-Scale Farms Through Stakeholders Collaboration. In: $12^{\text {th }}$ International Conference on Industrial Engineering and Industrial Management (In press)

3. Sutopo, W., Hisjam, M., Yuniaristanto: An Agri-food Supply Chain Model to Empower Farmers for Supplying Deteriorated Product to Modern Retailer. In: IAENG Transactions on Engineering Technologies: Special Issue of the International MultiConference of Engineers and Computer Scientists 2012, pp. 189--202. Dordrecht: Springer Netherlands (2013)

4. Sutopo, W., Hisjam, M., Yuniaristanto, Kurniawan, B.: A Goal Programming Approach for Assessing the Financial Risk of Corporate Social Responsibility Programs in Agri-food Supply Chain Network. Proceedings of the World Congress on Engineering 2013, pp. 732-$736(2013)$

5. Sutopo, W., Hisjam, M., Yuniaristanto: An Agri-food Supply Chain Model for Cultivating the Capabilities of Farmers Accessing Market Using Social Responsibility Program. International Scholarly and Scientific Research \& Innovation, 5(11), 1588--1592 (2011)

6. Sutopo, W., Hisjam, M., Yuniaristanto: An Agri-Food Supply Chain Model To Enhance the Business Skills of Small-Scale Farmers Using Corporate Social Responsibility. Makara Journal of Technology, 16(1), 43--50 (2012)

7. Sutopo, W., Hisjam, M., Yuniaristanto: Developing an Agri-Food Supply Chain Application for Determining the Priority of CSR Program to Empower Farmers as a Qualified Supplier of Modern Retailer. 2013 World Congress on Engineering and Computer Science, 1180--1184 (2013)

8. Wahyudin, R.S., Hisjam, M., Yuniaristanto, Kurniawan, B.: An Agri-food Supply Chain Model for Cultivating the Capabilities of Farmers in Accessing Capital Using Corporate Social Responsibility Program. Proceedings of the International MultiConference of Engineers and Computer Scientists, 877--882 (2015)

9. Jiménez, M., Arenas, M., Bilbao, A., Rodríguez, M.V.: Linear Programming with Fuzzy Parameters: An Interactive Method Resolution. Eur J Oper Res 177, 1599--1609 (2007)

10.Peidro, D., Mula, J., Jiménez, M., Botella, M.d.M.: A Fuzzy Linear Programming Based Approach for Tactical Supply Chain Planning in an Uncertainty Environment. Eur J Oper Res 205, 65--80 (2010)

11.Esteso, A., Alemany, M.M.E., Ortiz, A.: Conceptual Framework for Managing Uncertainty in a Collaborative Agri-Food Supply Chain Context. In: Working Conference on Virtual Enterprises. Springer, Cham, 715--724 (2017) 